\title{
Evaluación del potencial de aprendizaje en adultos mayores: influencia de la mediación en una prueba de memoria
}

\section{Learning potential assessment in the elderly: mediation influence in a memory assessment test}

\author{
María Josefina Gonzalez Aguilar ${ }^{12}$ \\ Milagros Rubio ${ }^{2}$ \\ Lina Grasso ${ }^{2}$ \\ ${ }^{1}$ Consejo Nacional de Investigaciones Científicas y Técnicas. Argentina \\ ${ }^{2}$ Centro de Investigaciones en Psicología y Psicopedagogía de la Universidad Católica Argentina
}

\begin{abstract}
Resumen: La versión "potencial de aprendizaje" de la Auditory Verbal Learning Test (AVLTPA) evalúa el rendimiento luego de una mediación activa por parte del evaluador. La AVLT-PA permite cuantificar una curva de aprendizaje mediante un puntaje de ganancia. Estudios previos demostraron que un puntaje de ganancia igual o mayor a 3 evidenciaría presencia de potencial de aprendizaje. Objetivos. Analizar diferencias en el rendimiento en la AVLT-PA en sujetos evaluados con mediación y sin mediación. Metodología. Se aplicó una batería neuropsicológica y la AVLT-PA a dos grupos de 35 adultos mayores cognitivamente sanos: un grupo recibió la mediación y el otro grupo no. Se comparó el rendimiento entre ambos grupos. Resultados. Se observaron diferencias en el último intento de la AVLT-PA, post-test y puntaje de ganancia en el grupo que recibió la mediación. Conclusiones. En ambos grupos, los sujetos inician la AVLT-PA recordando una cantidad de palabras similar. Sin embargo, los sujetos que recibieron la mediación del evaluador finalizan la prueba recordando más palabras que el grupo que no la recibió, evidenciando y validando la utilidad de la aplicación de esta versión de la prueba para la evaluación de potencial de aprendizaje.
\end{abstract}

Palabras clave: potencial de aprendizaje, memoria, evaluación dinámica, neuropsicología, envejecimiento, adultos mayores

\begin{abstract}
The "learning potential" version of the Auditory Verbal Learning Test (AVLT-LP) assesses memory performance after an active intervention (mediation). The AVLT-LP allows to quantify a learning curve by means of a gain score. Previous studies showed that if the gain score is equal- to or greater than 3 , it would be evidence of learning potential. Objectives. To analyze performance in healthy old adults assessed with AVLT-PA with mediation (MG) and without mediation (NoMG). Method. A neuropsychological battery was applied to 2 groups (MG and NoMG) of 35 cognitivelyhealthy older adults aged between 60 and 85 years old. The performance between both groups was compared. Results. The MG obtained higher scores than the NoMG in AVLT-LP's last trial, post-test and gain score. Conclusions. In both groups, the subjects started the AVLT-LP by remembering a similar number of words. However, the MG subjects retained more words than the NoMG showing the enhancement in memory applying the AVLT-PA to assess learning potential.
\end{abstract}

Keywords: learning potential, memory, dynamic assessment, neuropsychology, aging, elderly

Cómo citar este artículo:

Gonzalez Aguilar, M. J., Rubio, M., \& Grasso, L. (2018). Evaluación del potencial de aprendizaje en adultos mayores: influencia de la mediación en una prueba de memoria. Ciencias Psicológicas, 12(2), 215-222. doi: https://doi.org/10.22235/cp.v12i2.1684.

Correspondencia: María Josefina Gonzalez Aguilar, e-mail:josefina.gonzalezaguilar@hotmail.com; Milagros Rubio,e-mail:mili_r91@ hotmail.com; Lina Grasso, e-mail: lina_grasso1@gmail.com. Universidad Católica Argentina, Av. Alicia Moreau de Justo 1600, $2^{\circ}$ piso. Ciudad Autónoma de Buenos Aires - Argentina. 


\section{Introducción}

El potencial de aprendizaje se define como la distancia entre el nivel de desarrollo cognitivo actual de un individuo (su ejecución en determinada tarea) y el resultado que obtiene luego de aplicarse una mediación (Cabras, 2012; García, 2004). Esta definición se vincula estrechamente con el concepto de plasticidad cognitiva. La plasticidad cognitiva evidencia la capacidad de mejoría de un sujeto bajo condiciones óptimas de entrenamiento (Calero \& Navarro Gonzalez, 2006; Leon \& Roldan, 2016). Las evaluaciones realizadas desde esta perspectiva se conocen como "evaluaciones dinámicas" y se contraponen a las evaluaciones tradicionales consideradas "evaluaciones estáticas". Sternberg y Grigorenko (2003) sostienen que, existen tres principales diferencias entre estos dos abordajes. En primer lugar, mientras la evaluación estática observa el rendimiento actual de un sujeto de modo transversal (aptitudes preexistentes), la evaluación dinámica evalúa, además, si este rendimiento mejora bajo condiciones de entrenamiento, mediante la intervención o mediación de un experto (aptitudes potenciales involucradas en el aprendizaje). En segundo lugar, las evaluaciones estáticas y dinámicas se diferencian por la retroalimentación, ya que solo en las evaluaciones dinámicas se ofrece una retroalimentación o feedback de la performance del evaluado en el mismo momento de la evaluación. Por último, las pruebas estáticas y las dinámicas se diferencian por la actitud, el nivel de actividad y las intervenciones del evaluador: mientras que en las estáticas el evaluador procura no intervenir y ser neutral, en las dinámicas el evaluador busca vincularse e intervenir en el proceso de respuesta del sujeto (Sternberg \& Grigorenko, 2003).

El origen del constructo de potencial de aprendizaje se remonta al concepto de zona de desarrollo próximo de Vygotsky, quien definía el potencial de aprendizaje como la diferencia entre la habilidad de un individuo de resolver independientemente un problema y el nivel de desarrollo potencial que se da cuando resuelve ese problema con la colaboración de un experto (Vygotsky \& Cole, 1979). Mientras que la ejecución actual alude a las capacidades observadas de un sujeto, el potencial de aprendizaje aludiría a sus capacidades latentes (Malbrán \& Villar, 2002).

Las pruebas neurocognitivas que evalúan potencial de aprendizaje en el adulto mayor son, en general, pruebas estándar cuyas consignas origi- nales han sido modificadas para tal fin. Entre ellas, se encuentra la Batería de Evaluación de Potencial de Aprendizaje en Demencias -BEPAD- (Fernandez Ballesteros, Zamarrón, Tárraga, Moya, \& Iñiguez, 2003) que incluye cuatro pruebas clásicas de evaluación neuropsicológica (Lista Auditiva de Aprendizaje Verbal de Rey, Torre de Hanoi, Test de Posiciones y Fluencia Verbal) cuyas consignas se modificaron para que el evaluador introduzca una mediación, y evaluar el potencial de aprendizaje de un sujeto (Calero, 2000; Calero-García, 2004). Este tipo de evaluaciones buscan operacionalizar el concepto de aprendizaje evaluando el nivel inicial de rendimiento y la ejecución resultante luego de una mediación por parte del evaluador. Mediante estas consignas, el evaluador proporciona al sujeto ayudas para resolver una tarea (Cabras, 2012; Calero \& Navarro Gonzalez, 2006; Calero-García, 2004). La diferencia entre el rendimiento actual (previo a la mediación) y el potencial (luego de la mediación) se denomina puntaje de ganancia, y sería una medida del potencial de aprendizaje del evaluado. A través de la administración de este tipo de pruebas, la evaluación del potencial de aprendizaje permitiría valorar de modo clínico la presencia de plasticidad cognitiva en el adulto mayor (Calero \& Navarro Gonzalez, 2006).

Teniendo en cuenta que los cambios mnésicos en el envejecimiento han ocupado un papel destacado en las distintas investigaciones sobre adultos mayores, esta investigación privilegió como instrumento la versión de potencial de aprendizaje de la Lista Auditiva de Aprendizaje Verbal (Auditory Verbal Learning Test - AVLT) de Rey (1964), que evalúa memoria episódica. La evaluación neurocognitiva y las investigaciones sobre el envejecimiento han puesto el foco en la detección temprana de déficits cognitivos, especialmente en identificar marcadores que puedan resultar predictores de posible deterioro. Entre ellos puede encontrarse la alteración de la memoria episódica como posible predictor de evolución hacia una demencia de tipo Alzheimer (García Herranz, 2013).

En su versión original (Rey, 1964) la AVLT consta de una lista de 15 palabras sencillas (lista A) que el evaluador lee en voz alta solicitando al evaluado que repita las que recuerde. La lista A se lee 5 veces (A1 a A5), y se registran cuántas palabras el sujeto recordó en cada intento. La Auditory Verbal Learning Test - PA (AVLT-PA) es la adaptación de la AVLT tradicional para evaluar potencial de aprendizaje (Wiedl, Wienobost, y 
Schöttke, 1999; versión en castellano de Calero, 2000). En la AVLT-PA la lista A se presenta 6 veces (A1 a A6) y en los intentos intermedios (A3 y A4) se introduce la mediación o entrenamiento, que consta de un feedback del rendimiento del sujeto, y monitoreo y refuerzo de las palabras recordadas y a recordar. En la AVLT-PA, el promedio entre los primeros intentos (A1 y A2) se denomina pre-test, y el promedio entre los últimos intentos (A5 y A6) se denomina post-test. El pre-test se considera la línea de base de rendimiento del sujeto, y el post-test se considera la línea de rendimiento potencial, luego del entrenamiento. Luego se calcula la diferencia entre post-test y pre-test, y se obtiene un puntaje de ganancia. El puntaje de ganancia es la diferencia entre el rendimiento potencial y el rendimiento de base, y se interpreta como evidencia de plasticidad cognitiva. A diferencia de la versión estándar de la lista que requiere cierta neutralidad por parte del evaluador, en la AVLT-PA se exige que el evaluador se encuentre activamente implicado. La conducta que desarrolla el evaluador se adapta al rendimiento del sujeto, con el fin de estimular que el aprendizaje de las palabras se haga más evidente. De esta manera, se crea una relación de interacción entre ambos que construye una atmósfera de enseñanza y de ayuda personalizada (Cabras, 2012). En la tabla 1 se resume el procedimiento de administración en la AVLT-PA.

Estudios previos realizados por el equipo de Calero y Navarro Gonzalez en España concluyen que un puntaje de ganancia igual o mayor a 3 puntos hablaría de un "sujeto ganador", y sería evidencia válida de potencial de aprendizaje y plasticidad cognitiva (Calero \& Navarro González, 2006; Calero-García, 2004). En estos casos, se afirma que los sujetos ganadores son aquellos que han podido beneficiarse de la mediación. No debe dejar de mencionarse que en estos estudios se ha trabajado con muestras de adultos mayores con un bajo nivel educativo. En este sentido, el puntaje de corte de 3 puntos no es generalizable a todas las poblaciones y todos los niveles educativos, sino que deberían existir trabajos locales que confirmen cuál es el mejor punto de corte para considerar a un sujeto como ganador. En esta linea se inscribe el presente trabajo, como aporte local para el estudio del potencial de aprendizaje en adultos mayores latinoamericanos.

Calero y Navarro Gonzalez (2006) observaron que la mayoría de los adultos mayores que se encontraban en las fases iniciales de un proceso de declive cognitivo no presentaban potencial de aprendizaje (puntaje de ganancia bajo), mientras que los adultos mayores cognitivamente sanos aprendían significativamente más palabras en estas pruebas (puntaje de ganancia alto). Diversos trabajos han replicado este tipo de estudios en adultos mayores sanos, con deterioro cognitivo leve (DCL), y con distintos estadios de demencia (Boosman, Boven d'Eerdt, Visser-Meily, Nijboer, \& Heugten, 2016; Cassinello, Mestre, \& Fernández-Ballesteros, 2008; Fernández-Ballesteros, Zamarrón, \& Tárraga, 2005; Fernández-Ballesteros et al., 2003; Zamarrón, Mestre, \& Fernandez Ballesteros, 2009). En esta línea, la AVLT-PA sería de especial utilidad para el diagnóstico diferencial de DCL. Identificar marcadores neuropsicológicos que puedan resultar predictores de deterioro en estadios anteriores a una demencia resulta de gran interés para la investigación (García Herranz, 2013; Miranda et al., 2015; Russo et al., 2013).

En distintas investigaciones se ha observado la capacidad de los adultos mayores de beneficiarse de los entrenamientos y de la asistencia de un mediador (Calero \& Navarro Gonzalez, 2006; Francés, Barandiarán, Marcellán, \& Moreno, 2003; Calero-García, 2001; Navarro Gonzalez \& Calero, 2011). Sin embargo, los resultados de estos estudios no permiten saber si la ganancia se relaciona con la ayuda del mediador propiamente dicha, o si se

Tabla 1

Procedimiento de administración en la AVLT-PA(Calero y Navarro Gonzalez, 2006)

\begin{tabular}{|c|c|c|c|}
\hline Lista A & Tipo de consigna & Fase & \multirow{7}{*}{$\begin{array}{l}\text { Post-test }- \text { Pre-test }= \\
\text { Puntaje de ganancia }\end{array}$} \\
\hline A1 & Consigna estándar & \multirow{2}{*}{$\begin{array}{l}\text { Promedio A } 1 \text { y A } 2= \\
\text { Pre-test }\end{array}$} & \\
\hline A2 & Consigna estándar & & \\
\hline A3 & Consigna ampliada & \multirow{2}{*}{$\begin{array}{l}\text { Entrenamiento / } \\
\text { mediación }\end{array}$} & \\
\hline A4 & Consigna ampliada & & \\
\hline A5 & Consigna estándar & \multirow{2}{*}{$\begin{array}{l}\text { Promedio A5 y A6 = } \\
\text { Post-test }\end{array}$} & \\
\hline A6 & Consigna estándar & & \\
\hline
\end{tabular}


debe a la activación de habilidades ya presentes en los adultos mayores (Baltes, 1987; Navarro González \& Calero, 2011; Staudinger, Marsiske, $\&$ Baltes, 1995).

El propósito del presente estudio fue, por lo tanto, evaluar la influencia que tiene la mediación en una prueba de memoria episódica, y evaluar la utilidad de esta la versión de potencial de aprendizaje de esta clásica, y tan ampliamente utilizada, prueba de memoria. Asimismo, se propone una aproximación de la AVLT-PA con población sudamericana, dado que los estudios con este tipo de técnicas a nivel regional es muy escasa.

\section{Método}

\section{Participantes}

Participaron del estudio 70 adultos mayores argentinos cognitivamente sanos, con edades comprendidas entre los 60 y los 85 años $(X=$ 72.94; $D E=6.82)$ de ambos sexos $(67 \%$ de mujeres). La media de años de educación formal recibida de los participantes fue de 12.75 $(D E=3.96)$. Todos los sujetos fueron informados de los objetivos del estudio y firmaron voluntariamente un consentimiento informado.

\section{Instrumentos}

Los sujetos fueron evaluados por dos profesionales expertas en evaluación neuropsicológica en un encuentro de aproximadamente 1 hora y media. En el encuentro se administró una batería neuropsicológica que incluyó las siguientes pruebas:

- Mini Mental State Examination (MMSE) (Allegri et al., 1999; Butman et al., 2001; Folstein, Folstein, \& McHugh, 1975; Lobo et al., 1999): test utilizado para el rastreo inicial de deterioro cognitivo que permite evaluar las alteraciones de forma rápida y estandarizada. En el presente estudio se administró el MMSE versión rioplatense, de acuerdo con las instrucciones publicadas por el Grupo de Trabajo de Neuropsicología Clínica de la Sociedad Neurológica Argentina (Allegri et al.,1999, 2011).

- Frontal Assessment Battery-FAB - (Dubois, Slachevsky, Litvan, \& Pillon, 2000; Rodriguez-del Álamo, Catalán-Alonso, \& Carrasco-Marín, 2003): herramienta de screening para exploración de las funciones ejecutivas. La FAB evalúa de forma rápida y breve distintas hablidades cognitivas contemplados dentro de las funciones ejecutivas, como capacidad de conceptualización, flexibilidad mental, programación motora, sensiblidad a la interferencia, capacidad inhibitoria y autonomía ambiental.

- Test de Boston (Kaplan, Goodglass, \& Weintraub, 1983; Serrano et al., 2001): prueba de denominación de dibujos utilizada como instrumento para detectar alteraciones del lenguaje (anomias) y memoria semántica asociados al deterioro cognitivo en la Enfermedad de Alzheimer. En este estudio se utilizó la versión abreviada de 12 estímulos.

- Fluencia verbal (Carnero-Pardo \& LendínezGonzález, 1999; Labos, Trojanowski, del Rio, Zabala, \& Renato, 2013): test de evocación categorial donde se solicita al evaluado que en un lapso de 60 segundos denomine todas las palabras que pueda dentro de una categoría semántica (animales) y luego fonológica (que inicien con la letra $\mathrm{P}$ ). Se trata de una prueba sensible a la presencia de alteraciones cognitivas y procesos demenciales.

- Test del Reloj (Cacho, García-García, Arcaya, Vicente, \& Lantada, 1999; López, Allegri, \& Soto-Añari, 2014; Sunderland, Hill, Mellow, Lawlor, Gundersheimer, Newhouse, \& Grafman, 1989): prueba de rápida y sencilla administración, sensible para la detección de deterioro cognitivo. En este estudio se ha administrado el Test del Reloj a la orden, donde se solicita al evaluado que realice un reloj, coloque los números y que sus agujas marquen las 11:10.

- AVLT-PA (Calero \& Navarro-González, 2006): versión modificada de la AVLT para evaluar potencial de aprendizaje. En esta versión, el evaluador introduce la mediación en los ítems intermedios de la lista, y se obtiene un puntaje de ganancia tras calcular la diferencia entre los promedios de los últimos intentos y los primeros intentos. El procedimiento de administración de la AVLT-PA se detalló previamente en la tabla 1.

\section{Procedimiento}

Los participantes fueron divididos aleatoriamente en dos grupos de 35 sujetos: un grupo recibió la mediación en los intentos A3 y A4 de la AVLT$\mathrm{PA}$, y el otro grupo no recibió ninguna mediación o entrenamiento en dichos intentos. Las diferencias en el procedimiento de aplicación de la AVLT-PA según los grupos se describen en la tabla 2. Como se mencionó anteriormente, las consignas estándar consisten en la mera lectura de las palabras de la lista y el registro de las palabras recordadas por el sujeto, 
sin mayores intervenciones por parte del evaluador. Para las consignas ampliadas se siguieron los consignas de Calero y Navarro-González (2006), las cuales consisten, como se describió anteriormente, en la lectura de las palabras de la lista junto con una intervención activa del evaluador, aportando feedback de su rendimiento y un refuerzo en la lectura de las palabras que el sujeto aun no aprendió, registrando las palabras recordadas en cada intento.

En la tabla 3 se resumen las características sociodemográficas entre los grupos (con mediación y sin mediación), donde se observa que los mismos son homogéneos en términos de sexo, edad y nivel educativo.

\section{Análisis de datos}

El análisis constó en la comparación del rendimiento de los sujetos que recibieron la mediación y aquellos que no la recibieron, con la hipótesis de que los sujetos del grupo con mediación finalizarían la prueba recordando más palabras que aquellos que no recibieron la mediación. Con este fin, se procedió al análisis estadístico de comparación de medias mediante el cálculo de $t$ de Student para muestras independientes. Se calculó además el tamaño del efecto ( $d$ de Cohen) en las variables que presentaron diferencias significativas. Se utilizó el paquete estadístico SPSS v21.0.

\section{Resultados}

Se comparó el rendimiento de los dos grupos en las pruebas neuropsicológicas incluidas en la batería. Se observa que el rendimiento entre ambos grupos es similar en todas las pruebas administradas, salvo en el último intento de la AVLT-PA (ver tabla 4). Los puntajes obtenidos en pruebas neuropsicológicas clásicas como el Minimental $(p=.13)$, Test del reloj $(p=.33)$, fluencia verbal fonológica $(p=.67)$, fluencia verbal semántica $(p=.83)$, Test de Boston abreviado $(p=.19)$ y FAB $(p=.77)$ no presentaron diferencias entre los grupos. En términos de la AVLT-PA, los sujetos de ambos grupos

Tabla 2

Procedimiento de aplicación de la AVLT-PA según el grupo con mediación y sin mediación

Grupo con mediación $(\mathbf{N}=35)$

\begin{tabular}{|c|c|c|c|}
\hline & Tipo de consigna & & Puntajes \\
\hline Intento 1 & Estándar & Cant. de palabras recordadas & \multirow{2}{*}{$\begin{array}{l}\text { Puntaje Pre-test: Promedio de palabras recordadas en intentos } \\
1 \text { y } 2\end{array}$} \\
\hline Intento 2 & Estándar & Cant. de palabras recordadas & \\
\hline Intento 3 & Ampliada & Cant. de palabras recordadas & \multirow{2}{*}{ (El administrador realiza la mediación) } \\
\hline Intento 4 & Ampliada & Cant. de palabras recordadas & \\
\hline Intento 5 & Estándar & Cant. de palabras recordadas & \multirow{2}{*}{$\begin{array}{l}\text { Puntaje Post-test: Promedio de palabras recordadas en } \\
\text { intentos } 5 \text { y } 6\end{array}$} \\
\hline Intento 6 & Estándar & Cant. de palabras recordadas & \\
\hline \multicolumn{4}{|c|}{ Grupo sin mediación $(\mathrm{N}=35)$} \\
\hline & Tipo de consigna & & Puntajes \\
\hline Intento 1 & Estándar & Cant. de palabras recordadas & \multirow{2}{*}{$\begin{array}{l}\text { Promedio de palabras recordadas en intentos } 1 \text { y } 2 \text { : Puntaje } \\
\text { Pre-test }\end{array}$} \\
\hline Intento 2 & Estándar & Cant. de palabras recordadas & \\
\hline Intento 3 & Estándar & Cant. de palabras recordadas & \multirow{2}{*}{ (El administrador NO realiza la mediación) } \\
\hline Intento 4 & Estándar & Cant. de palabras recordadas & \\
\hline Intento 5 & Estándar & Cant. de palabras recordadas & \multirow{2}{*}{$\begin{array}{l}\text { Promedio de palabras recordadas en intentos } 5 \text { y } 6 \text { : Puntaje } \\
\text { Post-test }\end{array}$} \\
\hline Intento 6 & Estándar & Cant. de palabras recordadas & \\
\hline
\end{tabular}

Tabla 3

Características sociodemográficas de la muestra

\begin{tabular}{|c|c|c|c|c|c|c|c|c|}
\hline & \multicolumn{2}{|c|}{$\begin{array}{c}\text { Muestra } \\
\text { total } \\
\mathrm{N}=70\end{array}$} & \multicolumn{2}{|c|}{$\begin{array}{c}\text { Grupo con } \\
\text { mediación } \\
\mathrm{N}=35\end{array}$} & \multicolumn{2}{|c|}{$\begin{array}{c}\text { Grupo sin } \\
\text { mediación } \\
\mathrm{N}=35\end{array}$} & $t$ & $p$ \\
\hline \multirow[t]{2}{*}{ Sexo } & \multicolumn{2}{|c|}{$67 \%$ mujeres } & \multicolumn{2}{|c|}{$69 \%$ mujeres } & \multicolumn{2}{|c|}{$66 \%$ mujeres } & N/A & .87 \\
\hline & $X$ & d.t. & $X$ & d.t. & $X$ & d.t. & & \\
\hline Edad & 72.94 & 6.82 & 73.74 & 6.42 & 72.01 & 7.25 & .31 & .31 \\
\hline Nivel educativo & 12.75 & 3.96 & 12.49 & 3.83 & 13.07 & 4.15 & .56 & .56 \\
\hline
\end{tabular}


Tabla 4

Resultados obtenidos en las pruebas neuropsicológicas

\begin{tabular}{|c|c|c|c|c|c|c|c|}
\hline & \multirow{2}{*}{\multicolumn{2}{|c|}{$\begin{array}{l}\text { Grupo con } \\
\text { mediación } \\
\mathrm{N}=35\end{array}$}} & \multirow{2}{*}{\multicolumn{2}{|c|}{$\begin{array}{c}\text { Grupo sin } \\
\text { mediación } \\
\mathrm{N}=35\end{array}$}} & \multicolumn{2}{|c|}{$\begin{array}{c}\text { Prueba } t \text { de } \\
\text { Student }\end{array}$} & \multirow{3}{*}{$\begin{array}{c}\begin{array}{r}\text { Tamaño } \\
\text { del efecto }\end{array} \\
\mathrm{d} \\
\end{array}$} \\
\hline & & & & & $t$ & $p$ & \\
\hline & $X$ & $d t$ & $X$ & $d t$ & & & \\
\hline Minimental & 28.71 & 1.1 & 29.10 & .92 & -1.52 & .13 & $\mathrm{~N} / \mathrm{A}$ \\
\hline Test del reloj & 9.29 & 1.26 & 9.25 & 1.34 & -.98 & .33 & $\mathrm{~N} / \mathrm{A}$ \\
\hline Fluencia fonológica & 16.09 & 4.44 & 15.6 & 4.61 & .43 & .67 & $\mathrm{~N} / \mathrm{A}$ \\
\hline Fluencia semántica & 19.11 & 3.84 & 18.90 & 3.99 & .22 & .83 & $\mathrm{~N} / \mathrm{A}$ \\
\hline Test de Boston & 11.4 & .74 & 11.63 & .67 & -1.33 & .19 & $\mathrm{~N} / \mathrm{A}$ \\
\hline FAB & 17.23 & .88 & 17.17 & .79 & -.29 & .77 & $\mathrm{~N} / \mathrm{A}$ \\
\hline AVLT-PA Intento 1 & 4.49 & 1.72 & 4.73 & 1.48 & -.62 & .54 & $\mathrm{~N} / \mathrm{A}$ \\
\hline AVLT-PA Intento 2 & 7.14 & 1.99 & 7.5 & 1.86 & -.75 & .46 & $\mathrm{~N} / \mathrm{A}$ \\
\hline AVLT-PA Intento 3 & 9.37 & 2.16 & 8.63 & 2.14 & 1.38 & .17 & $\mathrm{~N} / \mathrm{A}$ \\
\hline AVLT-PA Intento 4 & 10.46 & 2.31 & 9.93 & 2.12 & .95 & .35 & $\mathrm{~N} / \mathrm{A}$ \\
\hline AVLT-PA Intento 5 & 11.29 & 2.37 & 10.23 & 2.78 & 1.65 & .1 & $\mathrm{~N} / \mathrm{A}$ \\
\hline AVLT-PA Intento 6 & 12.17 & 2.36 & 10.8 & 2.62 & 2.22 & .03 & .55 \\
\hline AVLT-PA Pre-test & 5.81 & 1.63 & 6.1 & 1.52 & -.73 & .47 & $\mathrm{~N} / \mathrm{A}$ \\
\hline AVLT-PA Post-test & 11.73 & 2.24 & 10.25 & 2.84 & 2.35 & .02 & .58 \\
\hline AVLT-PA Pje. de ganancia & 5.91 & 1.78 & 4.65 & 2.09 & 2.63 & .01 & .65 \\
\hline
\end{tabular}

Los valores se expresan como media $(X)$ y desvío estándar (d.t.)

inician recordando una cantidad de palabras similar ( $p>.05)$, pero aquellos que recibieron la mediación en los intentos 3 y 4 finalizan recordando significativamente más palabras que aquellos que no la recibieron. Se observa que las diferencias entre los puntajes se obtienen luego de la mediación del evaluador que se realiza en los intentos 3 y 4 , finalizando la prueba con diferencias estadísticamente significativas en el intento $6\left(t_{(68)}=2.22 ; p=.03\right)$, siempre a favor del grupo con mediación. Se observa un tamaño del efecto significativo de magnitud moderada para las diferencias entre los grupos en el intento $6(d=.55)$. La comparación del puntaje pre-test (promedio entre intentos 1 y 2) resultó en puntajes similares entre los dos grupos $(p=.47)$, pero el puntaje post-test (promedio entre intentos 5 y 6 ) evidencia resultados estadísticamente diferentes $\left(t_{(68)}=2.35 ; p=.02\right)$, indicando que aquellos sujetos que estuvieron expuestos a la mediación finalizaron la prueba con un rendimiento significativamente mejor que aquellos que no la recibieron. En este caso, también se observa un tamaño del efecto significativo de magnitud moderada para las diferencias entre los grupos en el puntaje de post-test $(d=.58)$. Finalmente, el hallazgo más significativo se da en el análisis de los puntajes de ganancia entre el grupo que recibió la mediación y el grupo que no la recibió. El grupo que recibió la mediación finalizó con un puntaje de ganancia promedio de 5.91 palabras $($ d.t. $=2.24)$, significativamente mayor $\left(t_{(68)}=2.63\right.$; $p=.01)$ que el grupo que no recibió mediación que finalizó con un puntaje de ganancia promedio de 4.65 palabras $($ d.t. $=2.09)$. El tamaño del efecto observado para estas diferencias es moderado a fuerte $(d=.65)$ y resulta el hallazgo más significativo del presente análisis. Resulta interesante que, sin bien la mayoría de los sujetos obtuvieron un puntaje de ganancia alto, cuando los sujetos son expuestos a la mediación por parte del evaluador, el puntaje de ganancia se dispara aún más.

\section{Discusión}

Los resultados obtenidos en el presente estudio sugieren, como se observaba en distintas investigaciones (Baltes \& Kliegl, 1992; Calero \& Navarro González, 2006), que al realizar una evaluación dinámica (con el uso de la mediación) los adultos mayores pueden mejorar su rendimiento cognitivo $\mathrm{y}$ beneficiarse de las intervenciones y ayudas de un experto evaluador. En nuestro estudio observamos que las intervenciones utilizadas fueron altamente significativas y lograron diferenciar a los sujetos que las recibieron de aquellos que no las recibieron.

La totalidad de los sujetos que fueron incluidos en el estudio fueron categorizados como cognitivamente sanos, y el rendimiento de estos en todas las pruebas se consideró normal. Sin embargo, el grupo de sujetos que recibió la mediación en la AVLT-PA presentó un rendimiento aún mejor en esta prueba 
que el grupo que no la recibió. Dado el exigente análisis realizado para corroborar la homogeneidad de los grupos en términos de variables sociodemográficas y rendimiento cognitivo, las diferencias en la AVLT-PA no puede explicarse por otro fenómeno que no sea la intervención del evaluador en la fase de la mediación. Ello indicaría que, ante condiciones de estimulación óptima, los adultos mayores evidencian un rendimiento cognitivo mayor.

Surge del análisis de los resultados que las muestran estaban compuestas por sujetos homogéneos en términos de sexo, edad, nivel educativo y rendimiento cognitivo, y las únicas diferencias se dan justamente en los ítems donde se vislumbra el efecto de la mediación por parte del evaluador. De ello puede deducirse que la inclusión de este tipo de consignas ampliadas que plantea la evaluación dinámica es significativa a la hora de evaluar el rendimiento potencial de un sujeto. Los resultados sugieren que la mediación resultó efectiva para mejorar el aprendizaje de una lista de palabras, ya que los valores muestran diferencias en la cantidad de palabras recordadas posterior a la mediación (post-test) pero no antes de la misma (pre-test). Esto resulta de particular interés dado que, hasta el momento, no se había evaluado separadamente la influencia concreta de la mediación en esta prueba de memoria en adultos mayores sanos (Calero \& Navarro González, 2006).

\section{Conclusión}

El objetivo de la evaluación dinámica es conocer, no solo el rendimiento observado, sino también el rendimiento potencial que podría alcanzar un sujeto si se lo expone a un ambiente enriquecedor y de estimulación. Autores que se apoyan en esta línea (Cabras, 2012; Calero \& Navarro Gonzalez, 2006; Calero-García, 2004; Malbrán \& Villar, 2002; Vygotsky \& Cole, 1979) conciben a la inteligencia como la capacidad de aprender, y buscan aproximarse a la comprensión del rendimiento de un sujeto considerando su rendimiento actual y su rendimiento potencial. De esta manera, las evaluaciones neurocognitivas que se sostienen en este marco teórico buscan medidas de potencial de aprendizaje y de plasticidad cognitiva. El propósito de esta metodología es operacionalizar el concepto de la curva de aprendizaje mediante la interpretación de un puntaje de ganancia.

Es interesante resaltar que las intervenciones realizadas en la fase de mediación de la AVLT-PA apuntaban simplemente a estimular al sujeto a que creara nuevas estrategias de aprendizaje, pero no se le dice explícitamente qué estrategia utilizar. Podría pensarse que, si las estrategias fueran mayores y más explícitas, los sujetos mejorarían aún más. En futuras investigaciones, evaluaremos el impacto de distintos tipos de intervenciones en esta prueba.

Este estudio asienta un antecedente de relevancia en la investigación del campo de la evaluación neuropsicológica dinámica. Se conoce que el aporte de este tipo de enfoque resulta valioso para la diferenciación diagnóstica entre un rendimiento dentro de parámetros normales y un rendimiento por debajo de lo esperable (Boosman, Boven d'Eerdt, Visser-Meily, Nijboer, \& Heugten, 2016; Calero-García, 2004). En este sentido, el objetivo de nuestra línea de investigación es continuar con la aplicación de la AVLT-PA tanto en adultos mayores sanos como con deterioro cognitivo.

La evaluación neurocognitiva dinámica y la observación del rendimiento no solo actual sino también potencial del adulto mayor demuestra ser un importante aporte a la descripción de la ejecución cognitiva y podría colaborar con un diagnóstico precoz más acertado de un deterioro cognitivo incipiente. El presente trabajo buscó aportar evidencias de la utilidad de la evaluación de potencial de aprendizaje y sus diferencias con la evaluación estática tradicional, con resultados contundentes que demostraron la validez de este tipo de enfoque. Nos encontramos en desarrollo de nuevos estudios donde evaluaremos el punto de corte del puntaje de ganancia que mejor explique este fenómeno, así como también nos encontramos estudiando la variabilidad de la plasticidad cognitiva a lo largo de las distintas trayectorias del envejecimiento y la variabilidad del rendimiento de la AVLT-PA en población clínica, principalmente con deterioro cognitivo leve y enfermedad de Alzheimer leve, con el objetivo de evaluar la capacidad diagnóstica y pronóstica de esta variable.

\section{Referencias}

Allegri, R.F., Ollari, J.A., Mangone, C.A., Arizaga, R.L., De Pascale, A., Pellegrini, M., Baumann, D., ...Taragano, F.E. (1999). El Mini-Mental State Examination en la Argentina: Instrucciones para su administración. Grupo de Trabajo de Neuropsicología: Sociedad Neurológica Argentina. Revista Neurológica Argentina, 24(1), 31-35.

Allegri, R.F., Arizaga, R.L., Bavec, C., Colli, L., Demey, I., Fernández, M.C., Frontera, S., ... Zuin, D. (2011). Guía de Práctica Clínica para la Enfermedad de Alzheimer. Neurología Argentina, 3, 120-37. doi: 0.1016/S1853-0028(11)70026-X 
Baltes, P. B. (1987). Theoretical propositions of life-span developmental psychology: On the dynamics between growth and decline. Developmental psychology, 23(5), 611-626. doi: 10.1037//0012-1649.23.5.611

Baltes, P. B., \& Kliegl, R. (1992). Further testing of limits of cognitive plasticity: Negative age differences in a mnemonic skill are robust. Developmental psychology, 28(1), 121-125. doi: 10.1037/0012-1649.28.1.121

Boosman, H., Bovend'Eerdt, T. J., Visser-Meily, J., Nijboer, T. C., $\&$ Heugten, C. M. (2016). Dynamic testing of learning potential in adults with cognitive impairments: A systematic review of methodology and predictive value. Journal of neuropsychology, 10(2), 186-210. doi: 10.1111/jnp.12063

Butman, J., Arizaga, R.L., Harris, P., Drake, M., Baumann, D., de Pascale, A., \& Ollari, J.A. (2001). El "Mini Mental State Examination" en Español. Normas para Buenos Aires. Rev Neurol Arg, 26(1), 11-15.

Cabras, E. (2012). Plasticidad cognitiva y deterioro cognitivo(Tesis de doctorado). Universidad Autónoma de Madrid, Madrid.

Cacho, J., García-García, R., Arcaya, J., Vicente, J. L., \& Lantada, N. (1999). Una propuesta de aplicación y puntuación del test del reloj en la enfermedad de Alzheimer. Rev Neurol, 28(7), 648-655.

Calero, M.D. (2000). Psicología de la vejez: el funcionamiento cognitivo. En Fernández-Ballesteros (Ed), Gerontología Social (201-228). Madrid: Ediciones Pirámide.

Calero, M. D. \& Navarro González, E. (2006). La plasticidad cognitiva en la vejez: Técnicas de evaluación e intervención. Barcelona: Octaedro.

Calero-García, M.D. (2001). Educación y funcionamiento cognitivo en mayores: aplicaciones del concepto de zona de desarrollo próximo. Tabanque: Revista pedagógica, (16), 157-168

Calero-García, M.D. (2004). Validez de la evaluación del potencial de aprendizaje. Psicothema, 16(2), 217-221.

Carnero-Pardo, C. \& Lendínez-González, A. (1999). Utilidad del test de fluencia verbal semántica para el diagnóstico de demencia. Revista de Nuerología, 29(8), 709-714.

Cassinello, M. D. Z., Mestre, L. T., \& Fernández-Ballesteros, R. (2008). Plasticidad cognitiva en personas con la enfermedad de Alzheimer que reciben programas de estimulación cognitiva. Psicothema, 20(3), 432-437.

Dubois, B., Slachevsky, A., Litvan, I., \& Pillon, B. (2000). The FAB: a frontal assessment battery at bedside. Neurology, 55, 1621-1626. doi: 10.1212/WNL.57.3.565

Fernández-Ballesteros, R., Zamarrón, M. D., \& Tárraga, L. (2005). Learning potential: a new method for assessing cognitive impairment. International Psychogeriatrics, 17(01), 119-128. doi: 10.1017/S1041610205000992

Fernández-Ballesteros, R., Zamarrón, M. D., Tárraga, L., Moya, R., \& Iñiguez, J. (2003). Cognitive plasticity in healthy, mild cognitive impairment (MCI) subjects and Alzheimer's disease patients: A research project in Spain. European Psychologist, 8(3), 148-159. doi: 10.1027//1016-9040.8.3.148

Folstein, M.F., Folstein, S.E., \& McHugh, P.R. (1975). Minimental state. A practical method for grading the cognitive state of patients for the clinician. J psychiatric Res, 12(3), 189-98. doi: 10.1016/0022-3956(75)90026-6

Francés, I., Barandiarán, M., Marcellán, T., \& Moreno, L. (2003). Estimulación psicocognoscitiva en las demencias. Anales del sistema sanitario de Navarra, 26(3), 383-403.

García Herranz, S. (2013). Relevancia de los test neuropsicológicos de evaluación de la memoria episódica y de la función ejecutiva como marcadores en la detección precoz del Deterioro Cognitivo Ligero (DCL): seguimiento longitudinal (tesis de doctorado). Universidad Nacional de Educación a Distancia, Madrid.

Kaplan, E.F., Goodglass, H., \& Weintraub, S. (1983) The Boston Naming Test. 2 ed. Philadelphia: Lea \& Febiger.

Labos, E., Trojanowski, S., del Rio, M., Zabala, K., \& Renato, A.
(2013). Perfiles de fluencia verbal en Argentina. Caracterización y normas en tiempo extendido. Neurol Arg, 5(2), 78-86. doi: 10.1016/j.neuarg.2013.04.005

León, I. \& Roldán-Tapia, L. (2016). Escala de Reserva Cognitiva y envejecimiento. Anales de psicología, 32(1), 218-223. doi: 10.6018/analesps.32.1.182331

Lobo, A., Saz, P., Marcos, G., Día, J.L., De la Cámara, C., Ventura, T., Morales-Asín, F., ... Lacámara, C. (1999). Revalidación y normalización del Mini-Examen Cognoscitivo (primera versión en castellano del Mini-Mental Status Examination) en la población general geriátrica. Medicina Clínica, 11, 767-74.

López N., Allegri R., \& Soto-Añari, M. (2014). Capacidad Diagnóstica y Validación Preliminar del Test del Reloj, Versión de Cacho a la Orden, para Enfermedad de Alzheimer de Grado Leve en Población Chilena. Revista Ecuatoriana de Neurología, 23,1-3.

Malbrán, M. C. \& Villar, C.M. (2002). La evaluación del potencial de aprendizaje: un procedimiento. Orientación y sociedad, 3, 1-14.

Miranda, A., Pruvost, M., Palau, F. G., Rimoldi, M. F., Viale, M., \& Cáceres, M. (2015). Perfiles neuropsicológicos: Enfermedad de Alzheimer y Parkinson, Deterioro cognitivo leve, Trastorno depresivo mayor y Envejecimiento. Cuadernos de Neuropsicología, 9(2), 30-48. doi: 10.7714/cnps/9.2.202

Navarro-González, E. \& Calero, M. D. (2011). Relación entre plasticidad y ejecución cognitiva: el potencial de aprendizaje en ancianos con deterioro cognitivo. European Journal of investigation in health, psychology and education, 1(2), 45-59. doi: 10.30552/ejihpe.v1i2.18

Rey, A. (1964). L'examen clinique en psychologie. Paris: Presse Universitaires de France.

Rodriguez-del Álamo, A., Catalán-Alonso, M.J., \& CarrascoMarín, L. (2003). FAB: Aplicación preliminar española de la batería neuropsicológica de funciones ejecutivas a 11 grupos de pacientes. Rev Neurol, 36(7), 605-608.

Russo, M. J., Campos, J., Martin, M. E., Clarens, M. F., Sabe, L., \& Allegri, R. F. (2013). Índice de discriminabilidad en memoria de reconocimiento en deterioro cognitivo leve amnésico y demencia tipo Alzheimer leve. Un estudio preliminar. Neurología Argentina, 5(4), 241-249. DOI: 10.5579/rnl.2017.0317

Serrano, C., Allegri, R., Drake, M., Butman, J., Harris, P., Nagle, C., \& Ranalli, C. (2001). Versión abreviada en español del test de denominación de Boston: su utilidad en el diagnóstico diferencial de la enfermedad de Alzheimer. Revista de Neurología, 33(7), 624-627.

Staudinger, U. M., Marsiske, M., \& Baltes, P. B. (1995). Resilience and reserve capacity in later adulthood: Potentials and limits of development across the life span. Developmental psychopathology, 2, 801-847. doi: 10.1017/ S0954579400006155

Sternberg, R.J. \& Grigorenko, E.L. (2003). Evaluación dinámica. Naturaleza y medición del potencial de aprendizaje. Buenos Aires: PAIDOS.

Sunderland, T., Hill, J.L., Mellow, A.M., Lawlor, B.A., Gundersheimer, J., Newhouse, P.A., \& Grafman, J.H. (1989). Clock drawing in Alzheimer's disease. A novel measure of dementia severity. Journal of the American Geriatric Society, 37(8),725-9. doi: 10.1111/j.1532-5415.1989.tb02233.x

Vigotsky, L. S. \& Cole, M. (1979). El desarrollo de los procesos psicológicos superiores. Barcelona: Crítica.

Wiedl, K.H., Wienobost, J., \& Schöttke, H. (1999). Estimating rehabilitation potential in schizophrenic subjects. En Brenner, H.D., Boker, W. y Genners, R. (Eds). The treatment of schizophrenia: Status and emerging trends. Bonn: Hogrefe and Huber.

Zamarrón, M. D., Mestre, L. T., \& Ballesteros, R. F. (2009). Cognitive plasticity in Alzheimer's disease patients receiving cognitive stimulation programmes. Psychology in Spain, (13), 48-54. 